\title{
Unidentified Infrared Discrete Emission Bands
}

\author{
Vladimir S. Netchitailo \\ Biolase Inc., Irvine, USA \\ Email: netchitailov@gmail.com
}

How to cite this paper: Netchitailo, V.S. (2022) Unidentified Infrared Discrete Emission Bands. Journal of High Energy Physics, Gravitation and Cosmology, 8, 243-253. https://doi.org/10.4236/jhepgc.2022.82018

Received: December 16, 2021

Accepted: February 22, 2022

Published: February 25, 2022

Copyright $\odot 2022$ by author(s) and Scientific Research Publishing Inc. This work is licensed under the Creative Commons Attribution International License (CC BY 4.0).

http://creativecommons.org/licenses/by/4.0/

\begin{abstract}
Unidentified Infrared emission bands (UIBs) are infrared discrete emissions from circumstellar regions, interstellar media (ISM), star-forming regions, and extragalactic objects for which the identity of the emitting materials is unknown. The main infrared features occur around peaks at 3.3, 6.2, 7.7, 8.6, 11.2 , and $12.7 \mu \mathrm{m}$ with the photon's rest energy at the peaks $0.376,0.200$, $0.161,0.144,0.111$, and $0.098 \mathrm{eV}$, respectively. The UIB emission phenomenon has been studied for about forty five years. The prevailing hypothesis is that the materials responsible for UIB are polycyclic aromatic hydrocarbon (PAH) molecules. PAHs are thought to be one of the main forms in which carbon exists in space. And yet, not a single member of this group of compounds had been identified in space definitively until now [1]. In frames of Hypersphere World-Universe Model (WUM), we introduced Dark Matter (DM) particles, named DIONs, with the rest energy $0.199 \mathrm{eV}$ and an energy density of $68.8 \%$ of the total energy density of the World. DIONs compose Outer shells of DM Supercluster's Cores-the main objects of the World [2]. In this paper, we give an explanation of UIB emission based on the self-annihilation of DM particles DIONs and biDIONs (DIONs pairs) with a rest energy about $0.38 \mathrm{eV}$ that depends on the binding energy. To the best of our knowledge, WUM is the only cosmological model in existence that is consistent with UIB emission phenomenon.
\end{abstract}

\section{Keywords}

Hypersphere World-Universe Model, Law of Energy Conservation, Interstellar Media, Dark Matter Particles Self-annihilation, Unidentified Infrared Emission Bands

\section{Introduction}

In the present manuscript, which considers the phenomenon "Unidentified Infrared Discrete Emission Bands", we: 
- Review articles published in this area for forty five years by hundreds of scientists who proposed the prevailing hypothesis that the materials responsible for UIB are polycyclic aromatic hydrocarbon (PAH) molecules. And yet, not a single member of this group of compounds had been identified in space definitively until now [1];

- Give a principally different explanation of UIB emission based on the self-annihilation of DM particles DIONs and biDIONs (DIONs pairs) that is consistent with the law of the energy conservation;

- Do not pretend on the development of a theory of this phenomenon in frames of WUM.

The history of the Unidentified Infrared Emission Bands (UIBs) can be traced back to at least 1977. In 2016, S. Kwok provided an excellent review of this phenomenon [3]:

A family of unidentified infrared emission (UIE) features at 3.3, 6.2, 7.7, 8.6, and $11.3 \mu \mathrm{m}$ was discovered almost 40 years ago [4]. The $3.3 \mu \mathrm{m}$ feature was first identified as the $C-H$ stretching mode of aromatic compounds by $R$. F. Knacke in 1977 [5]. In 1981, the organic affiliation was extensively discussed by W. W. Duley and D. A. Williams who assigned the 3.3 and $11.3 \mu \mathrm{m}$ features to graphitic (aromatic) materials. Specifically, the $3.3 \mu \mathrm{m}$ feature is identified as due to aromatic $C$ - $H$ stretch, the $6.2 \mu \mathrm{m}$ feature as aromatic $C$-C stretch, the $8.6 \mu \mathrm{m}$ feature as the $C$ - $H$ in-plane bending mode, and the 11.3, 12.4 and 13.3 $\mu \mathrm{m}$ features as due to solo, duo, and trio $\mathrm{C}-\mathrm{H}$ out-of-plane bending modes, respectively [6].

In 1980s, astronomers went on to hypothesize that the materials responsible for Unidentified Infrared emission (UIR) should be polycyclic aromatic hydrocarbon (PAH) molecules, which are chemical compounds containing only carbon and hydrogen - that is composed of multiple aromatic rings [7] [8] [9]. PAHs are prevalent in Interstellar Media (ISM) of galaxies in both the nearby and distant Universe and make up a dominant emission mechanism in the mid-infrared wavelength range, containing as much as $10 \%$ of the total integrated infrared luminosity of galaxies [Wikipedia. Polycyclic aromatic hydrocarbon].

J. P. Simpson, et al. have taken spectra of many positions in the central $25 \mathrm{~min}$ of the Galactic Center. The spectra exhibit strong UIR/PAH features at 6.2, 7.7, 8.6 and 11.3 microns, in addition to the ionic lines of (Ne II), at 12.8 microns, (S III) 18.7 microns, and (Ar II) 6.98 microns. Compared to the spectrum of the Orion nebula, the Galactic Center spectra have similar PAH features, but the Orion Nebula also has strong lines of (He III) 15.6 microns, (S IV) 10.5 microns, and (Ar III) 8.99 microns [10].

D. Cesarsky, et al. present mid-infrared spectro-imagery and high-resolution spectroscopy of the Orion bar and of a region in the Orion nebula (see Figure 1). Their data shows emission from amorphous silicate grains from the entire $\mathrm{H}$ II region and around the isolated star Theta2 Ori A. In their opinion, the observed spectra can be reproduced by a mixture of interstellar silicate and carbon 


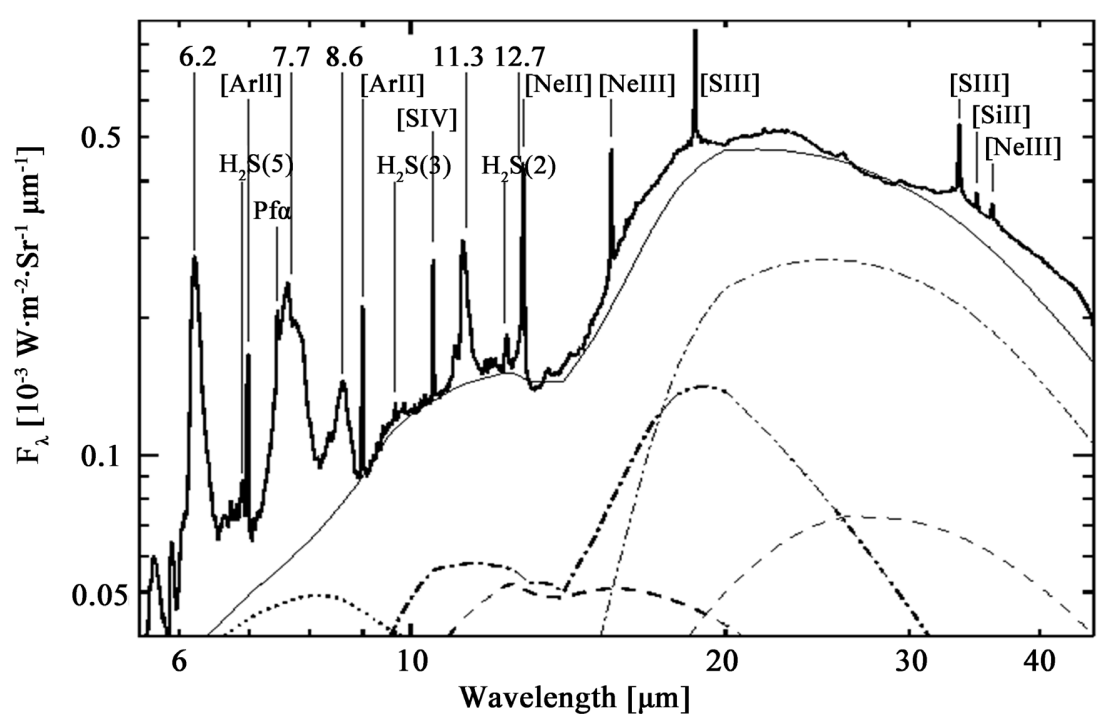

Figure 1. Short Wavelength Spectrometer (SWS) spectrum (full line) in the Orion nebula. The identification of the strongest spectral features $(6.2,7.7,8.6,11.3$, and $12.7 \mu \mathrm{m})$ is indicated. Adapted from [11].

grains heated by the radiation of the hot stars present in the region [11].

In 2008, A. Tielens provided an excellent review of Interstellar PAH Molecules [12]: Large polycyclic aromatic hydrocarbon $(P A H)$ molecules carry the infrared (IR) emission features that dominate the spectra of most galactic and extragalactic sources. This review surveys the observed mid-IR characteristics of these emission features and summarizes laboratory and theoretical studies of the spectral characteristics of PAHs and the derived intrinsic properties of emitting interstellar PAHs. Dedicated experimental studies have provided critical input for detailed astronomical models that probe the origin and evolution of interstellar PAHs and their role in the universe. The physics and chemistry of PAHs are discussed, emphasizing the contribution of these species to the photoelectric heating and the ionization balance of the interstellar gas and to the formation of small hydrocarbon radicals and carbon chains. Together, these studies demonstrate that PAHs are abundant, ubiquitous, and a dominant force in the interstellar medium of galaxies.

In 2013, S. Kwok and Y. Zhang asked the following question: "Why is no PAH molecule detected?" [13]:

Since the UIR features are widely observed throughout the Galaxy and represent up to $20 \%$ of the total energy output of starburst galaxies, the carrier of these features must be extremely common and abundant. PAH have very strong absorption in the $U V(0.12-0.3 \mu \mathrm{m})$. If the abundance of $P A H$ is high enough to emit strongly in the infrared, then it is difficult to understand why the same molecules in the ground electronic state will not cause absorption features in the $U V$. Sensitive searches have been made in the UV with the Hubble Space Telescope and the Very Large Telescope, but no detection was made, putting very low upper limits on the abundance of PAH molecules in the diffuse interstellar 
medium (ISM). The absence of UV absorption features of the diffuse ISM is in fact a strong argument against a molecular carrier $P A H$.

S. Kwok and Y. Zhang suggested that the carrier of UIR bands is an amorphous carbonaceous solid with mixed aromatic/aliphatic structures, rather than free-flying polycyclic aromatic hydrocarbon molecules. Through spectral fittings of the astronomical spectra of UIR bands, the authors showed that a significant amount of the energy is emitted by aliphatic component, implying that aliphatic groups are an essential part of the chemical structure [13].

In 2021, D. Bradley made the following conclusion about PAH molecules: Interstellar spectra recorded using mid-infrared spectroscopy show many emission lines. The spectral lines are usually assigned to polycyclic aromatic hydrocarbons (PAHs). Indeed, PAHs are thought to be one of the main forms in which carbon exists in space. And yet, not a single member of this group of compounds had been identified in space definitively until now [1].

\section{Dark Matter in WUM}

Hypersphere World-Universe Model (WUM) is based on two parameters only $\alpha$ and $Q[14]$ :

- $\alpha$ is the dimensionless Rydberg constant: $\alpha=\left(2 a R_{\infty}\right)^{1 / 3} \quad$ (that was later named Fine-structure constant) and $a$ is a basic unit of size $a=1.7705641 \times 10^{-14} \mathrm{~m}$;

- $Q$ is the dimensionless time-varying quantity that is a measure of the Size $R$ and Age $A_{r}$ of the World:

$$
Q=\frac{R}{a}=\frac{A_{\tau}}{t_{0}}
$$

which in present epoch equals to: $Q=0.759972 \times 10^{40}$ and is, in fact, the Dirac Large Number;

- A basic unit of time $t_{0}$ equals to: $t_{0}=a / c=5.9059662 \times 10^{-23} \mathrm{~s}$ and $c$ is the Gravitodynamic constant that is the ratio of the absolute gravitomagnetic unit of charge $E_{0}$ to the absolute gravitostatic unit of charge $E_{0} / c$ ( $E_{0}=h c / a$ is a basic unit of energy and $h$ is Planck constant).

It is worth noting that the Speed of light in vacuum, commonly denoted as $\mathcal{C}$, is not related to the World in our Model, because there is no vacuum in it. Instead, there is the Medium of the World consisting of elementary particles.

The prospect that Dark Matter Particles (DMPs) might be observed in Centers of Macroobjects has drawn many new researchers to the field in the last forty five years. In 1977-1980, indirect effects in cosmic rays and gamma-ray background from the annihilation of Cold DM in the form of heavy stable neutral leptons in Galaxies were considered in pioneer articles [15]-[20]. Self-annihilation of DMPs is the cornerstone of WUM.

WUM proposes multicomponent DM system consisting of two couples of coannihilating Dark Matter Particles (DMPs): a heavy Dark Matter Fermion (DMF)-DMF1 $(1.3 \mathrm{TeV})$ and a light spin-0 boson-DIRAC $(70 \mathrm{MeV})$ that is a dipole of Dirac's monopoles with charge $\mu=e / 2 \alpha$ ( $e$ is the elementary 
charge); a heavy fermion-DMF2 (9.6 GeV) and a light spin-0 boson-ELOP $(340 \mathrm{keV})$ that is a dipole of preons with electrical charge e/3; self-annihilating fermions - DMF3 (3.7 keV) and DMF4, named DION, (0.2 eV).

WUM postulates that rest energies of DMFs and bosons are proportional to a basic unit of energy $E_{0}$ multiplied by different exponents of $\alpha$ and can be expressed with the following formulae [14]:

$\begin{array}{ll}\text { DMF1 (fermion): } & E_{\text {DMF1 }}=\alpha^{-2} E_{0}=1.3149950 \mathrm{TeV} \\ \text { DMF2 (fermion): } & E_{\text {DMF2 }}=\alpha^{-1} E_{0}=9.5959823 \mathrm{GeV} \\ \text { DIRAC (boson): } & E_{\text {DIRAC }}=\alpha^{0} E_{0}=70.025267 \mathrm{MeV} \\ \text { ELOP (boson): } & E_{\text {ELOP }}=2 / 3 \alpha^{1} E_{0}=340.66606 \mathrm{keV} \\ \text { DMF3 (fermion): } & E_{\text {DMF3 }}=\alpha^{2} E_{0}=3.7289402 \mathrm{keV} \\ \text { DION (fermion): } & E_{\text {DION }}=\alpha^{4} E_{0}=0.19857111 \mathrm{eV}\end{array}$

Note that the rest energy of electron $E_{e}$ equals to: $E_{e}=\alpha E_{0}$ and the Rydberg unit of energy is: $R y=h c R_{\infty}=0.5 \alpha^{3} E_{0}=13.605693 \mathrm{eV}$.

We still do not have a direct confirmation of DMPs' rest energies, but we do have various indirect observations. The signatures of DMPs self-annihilation with expected rest energies of $1.3 \mathrm{TeV}$; $9.6 \mathrm{GeV}$; $70 \mathrm{MeV}$; $340 \mathrm{keV}$; $3.7 \mathrm{keV}$ are found in spectra of the diffuse gamma-ray background and the emissions of various Macroobjects in the World. We connect observed gamma-ray spectra with the structure of Macroobjects (nuclei and shells composition). Self-annihilation of those DMPs can give rise to any combination of gamma-ray lines. Thus, the diversity of Very High Energy gamma-ray sources in the World has a clear explanation in WUM [21].

It is worth recalling a story about neutrinos: "The neutrino was postulated first by W. Pauli in 1930 to explain how beta decay could conserve energy, momentum, and angular momentum (spin). But we still don't know the values of neutrino masses". Although we still cannot measure neutrinos' masses directly, no one doubts their existence.

According to WUM, the total DIONs energy density in the World $68.8 \%$ is almost ten times greater than the total baryonic energy density $7.2 \%$. The rest $24 \%$ is the energy density of five DM particles above. At such a high DIONs concentration, "DIONs pairs" (biDIONs) can be created. Their concentration may indeed be sufficient to undergo the self-annihilation, and as a result create an emission with $3.3 \mu \mathrm{m}$. In the present paper we discuss the possibility to explain UIB emission features through self-annihilation of DIONs and biDIONs with the rest energy of about $0.38 \mathrm{eV}$.

\section{Weak Interaction}

According to WUM, strength of gravity is characterized by the gravitational parameter [2]:

$$
G=G_{0} \times Q^{-1}
$$

where $G_{0}=a^{2} c^{4} / 8 \pi h c$ is an extrapolated value of $G$ at the Beginning of the World $(Q=1)$. The range of the gravity equals to the size of the World $R$ : 


$$
R=a \times Q=1.34558 \times 10^{26} \mathrm{~m}
$$

In WUM, weak interaction is characterized by the parameter $G_{W}$ :

$$
G_{W}=G_{0} \times Q^{-1 / 4}
$$

which is about thirty orders of magnitude greater than $G$. The range of the weak interaction $R_{W}$ in the present Epoch equals to:

$$
R_{W}=a \times Q^{1 / 4}=1.65314 \times 10^{-4} \mathrm{~m}
$$

that is much greater than the range of the weak nuclear force. The weak interaction between DMPs will be efficient in case when their concentration $n_{W}$ is larger than

$$
n_{W}^{c r}=2.21346 \times 10^{11} \mathrm{~m}^{-3}
$$

The calculated maximum average concentration of DIONs in the largest shell of Superclusters $n_{D I O N}^{S C}$ is [2]:

$$
n_{D I O N}^{S C} \cong 4.2 \times 10^{15} \mathrm{~m}^{-3}
$$

It shows that a distance between particles is around $\sim 10^{-5} \mathrm{~m}$, which is much smaller than $R_{W}$. Thus, the introduced weak interaction of DMPs with Matter will provide integrity of all Shells in DM Cores of all Macroobjects. In our view, weak interaction between DM particles DMF3 provides integrity of Fermi Bubbles and Solar Corona [2].

\section{Galaxies in WUM}

According to WUM, the total energy density of the World $\rho_{W}$ equals to $\rho_{W}=3 \rho_{0} \times Q^{-1}$ throughout the World's evolution, where a basic unit of energy density $\rho_{0}$ is: $\rho_{0}=E_{0} / a^{3}$. The energy density of the Medium is two-thirds of the total energy density and Macroobjects (Superclusters, Galaxies, and Extrasolar systems) - one-third in all cosmological times. The calculated average energy density of DIONs in the Intergalactic Medium $\rho_{D I O N}^{I G M}$ is:

$$
\rho_{\text {DION }}^{I G M}=0.688 \times 2 \rho_{0} \times Q^{-1}
$$

and the calculated average concentration of them $n_{D I O N}^{I G M}$ is:

$$
n_{D I O N}^{I G M}=1.15 \times 10^{10} \mathrm{~m}^{-3}
$$

that is much smaller than $n_{W}^{c r}$. It means that the weak interaction between DIONs is not efficient, the self-annihilation between them is practically not happening, and hence, UIB emission in the Intergalactic Medium is not observed. This result is in good agreement with the experimental observations (see Section 2).

Milky Way (MW) is the second-largest spiral galaxy in the Local Group (after Andromeda Galaxy), with its estimated visible stellar disk diameter

$$
D_{M W}=185 \pm 15 \mathrm{kly}
$$

thickness of thin stellar disk about 2 kly and mass 


$$
M_{M W}=(1.6-3.2) \times 10^{42} \mathrm{~kg}
$$

In our view, $\mathrm{MW}$ is a Disk Bubble (DB) whose boundary with the Intergalactic Medium has a surface energy density $\sigma_{0}$ that is a basic unit of surface energy density: $\sigma_{0}=E_{0} / a^{2}$. The Disk Bubble contains Interstellar Medium (ISM) and (100 - 400) billion Extrasolar systems. According to WUM, mass of MW equals to:

$$
M_{M W}=\frac{\pi D_{M W}^{2} \sigma_{0}}{2 c^{2}}
$$

We calculate $D_{M W}$ by the following equation:

$$
D_{M W}=\left(\frac{2 M_{M W} c^{2}}{\pi \sigma_{0}}\right)^{1 / 2}=(170-240) \mathrm{kly}
$$

The calculated value of the visible stellar disk diameter is in good agreement with its estimated value obtained by astronomers. Considering the average stellar disk diameter $D_{M W}=185$ kly we can calculate the mass of MW:

$$
M_{M W}=1.92 \times 10^{42} \mathrm{~kg}
$$

average energy density:

$$
\rho_{M W}=3.78 \times 10^{-3} \mathrm{~J} \cdot \mathrm{m}^{-3}
$$

and average concentration of DIONs in ISM $n_{D I O N}^{I S M}$ :

$$
n_{\text {DION }}^{I S M}=5.45 \times 10^{16} \mathrm{~m}^{-3}
$$

that is over five orders of magnitude larger than the critical concentration $n_{W}^{c r}$. Density fluctuations of them could happen in ISM and clumps of DIONs will arise with a density that is sufficient for their self-annihilation. Concentration of DIONs in circumstellar regions, star-forming regions, and extragalactic objects is even larger than $n_{D I O N}^{I S M}$. At such a high DIONs concentration, "DIONs pairs" (biDIONs) can be created, their concentration $n_{\text {biDION }}^{I S M}$ may indeed be sufficient to undergo the weak interaction and self-annihilation $\left(n_{b i D I O N}^{I S M} \geq n_{W}^{c r}\right)$, and as a result, UIB emission at $3.3 \mu \mathrm{m}$ can be observed inside of MW.

Andromeda Galaxy (AG) is a barred spiral galaxy approximately $2.5 \mathrm{Mly}$ from Earth and the nearest large galaxy to MW with about $10^{12}$ stars. It has an estimated visible stellar disk diameter $\sim 220 \mathrm{kly}$ and mass $(3 \pm 1) \times 10^{42} \mathrm{~kg}$. Parameters of AG are close enough to the parameters of MW. It seems reasonable that the calculations of the galaxy parameters made above for MW are valid for AG also.

The experimental observations of galaxies in the universe showed that most of them are disk galaxies: about $60 \%$ are ellipticals and about $20 \%$ are spirals [22]. Considering the fact that the calculated concentrations of DIONs are about five orders of magnitude larger than the critical concentration, we can suppose that our conclusions for MW are fair for all galaxies in the World.

It is worth noting that in frames of WUM the Interstellar Medium in Galaxies is, in fact, a low viscosity "Liquid" with "Weak bonds" between DM particles 
DIONs, which are constantly moving concerning each other, and the weak bonds are continually breaking and reforming. This "Liquid" can be named "Aether." In WUM, Galaxies emerged due to the Explosive Volcanic Rotational Fission of Overspinning DM Superclusters' Cores with the biggest Shells composed of DIONs. As the result of this mechanism, the Galaxy Bubbles created, which have look like "Chicken egg" at that time:

- "Yolk" - spinning liquid DM Core of galaxy with high viscosity composed of DMPs (DMF1, DMF2, and DMF3);

- "Albumen"-liquid ISM with low viscosity made of DIONs with dissolved other DMPs;

- "Membrane"-boundary between ISM and Intergalactic Medium with surface energy density $\sigma_{0}$.

"Yolk" contains one-third of the total galaxy Matter, and "Albumen" adds up to two-thirds of the total galaxy Matter. Extrasolar systems emerge due to the Explosive Volcanic Rotational Fission of Overspinning DM galaxy Core and enter ISM. As the result, galaxy Bubble expands in the plane of the spinning Core and becomes a Disk Bubble.

\section{Explanation of Unidentified Infrared Emission Bands}

It is well-known that electron-positron annihilation is a fundamental process when an electron and a positron collide in free space. The energy-momentum conservation requires that the annihilation takes place with the emission of at least two photons. Annihilation of electron-positron producing more than two photons is less likely. Annihilation of an electron-positron pair accompanied by emission of three photons is discussed by A. Ore and J. L. Powell who calculated the cross section for this process. They found that the ratio of the calculated cross section to that of ordinary two-photon annihilation is 1:370 [23].

The main infrared features of UIBs occur around peaks at 3.3, 6.2, 7.7, 8.6, 11.2 , and $12.7 \mu \mathrm{m}$ with the photon's rest energy at the peaks $0.376,0.200,0.161$, $0.144,0.111$, and $0.098 \mathrm{eV}$, respectively. These UIBs are observed in ISM of galaxies in both the nearby and distant World and are, in fact, the "Fingerprints" of all galaxies.

By analogy with the electron-positron annihilation, we suppose that DIONs and biDIONs self-annihilate with the emission of two or three photons. Indeed, the self-annihilation of them follows an energy conservation requirement:

- Two biDIONs with a rest energy about $0.38 \mathrm{eV}$ can produce two photons with the rest energy $0.376 \mathrm{eV}(3.3 \mu \mathrm{m})$;

- Two DIONs with the rest energy $0.199 \mathrm{eV}$ can produce two photons with the rest energy $0.200 \mathrm{eV}(6.2 \mu \mathrm{m})$;

- Two DIONs with the rest energy $0.199 \mathrm{eV}$ can produce three photons with the rest energies $0.161 \mathrm{eV}(7.7 \mu \mathrm{m}), 0.144 \mathrm{eV}(8.6 \mu \mathrm{m})$, and $0.111 \mathrm{eV}(11.2$ $\mu \mathrm{m})$, or $0.098 \mathrm{eV}(12.7 \mu \mathrm{m})$;

- Two DIONs with the rest energy $0.199 \mathrm{eV}$ can produce three photons with 
the rest energies $0.200 \mathrm{eV}(6.2 \mu \mathrm{m}), 0.111 \mathrm{eV}(11.2 \mu \mathrm{m})$, and $0.098 \mathrm{eV}(12.7$ $\mu \mathrm{m})$.

The following results speak in favor of this explanation:

- The source of UIBs is prevalent in ISM of all galaxies and makes up a dominant emission mechanism in the mid-infrared wavelength range, containing as much as $10 \%$ of the total integrated infrared luminosity of galaxies and up to $20 \%$ of the total energy output of starburst galaxies;

- All studies of this phenomenon demonstrate that the source of UIBs is extremely common, abundant, ubiquitous, and a dominant force in ISM of galaxies;

- UIBs are the same infrared discrete emissions from various regions of Galaxies: ISM, circumstellar regions, star-forming regions, extragalactic objects, and regions of cold molecular gas, for which the identity of the emitting materials is unknown;

- The spectra of the Galactic Center exhibit strong UIB features at 6.2, 7.7, 8.6 and 11.3 microns;

- The absence of UV absorption features of the diffuse ISM is, in fact, a convincing argument against a group of $\mathrm{PAH}$ molecules as the carrier of UIBs. Not a single member of this group of compounds had been identified in space definitively until now.

WUM does not attempt to explain all available cosmological data, as that is an impossible feat for any one article. Nor does WUM pretend to have built an all-encompassing theory that can be accepted as is. According to Reviewer, the document so engendered is a gateway to a new testing program, which may lead to falsifiable results.

\section{Acknowledgements}

I am very grateful to the anonymous Reviewer who understands the positive merits of my new explanation but requires in the future that articles submitted using this theory do full explanations, due to the novelty of this approach. His useful comments and suggestions have led to an overall improvement of the manuscript. Special thanks to my son Ilya Netchitailo who helped me refine the Model and improve its understanding.

\section{Conflicts of Interest}

The author declares no conflicts of interest regarding the publication of this paper.

\section{References}

[1] Bradley, D. (2021) Interstellar Polycyclic Aromatic Hydrocarbons. Wiley-VCH $\mathrm{GmbH}$, Hoboken.

https://www.chemistryviews.org/details/ezine/11294148/Interstellar Polycyclic Aro matic Hydrocarbons.html

https://doi.org/10.1002/chemv.202100027 
[2] Netchitailo, V.S. (2021) Decisive Role of Dark Matter in Cosmology. Journal of High Energy Physics, Gravitation and Cosmology, 8, 115-142.

https://vixra.org/pdf/2110.0166v1.pdf https://doi.org/10.4236/jhepgc.2022.81009

[3] Kwok, S. (2016) Complex Organics in Space from Solar System to Distant Galaxies. The Astronomy and Astrophysics Review, 24, 8.

https://doi.org/10.1007/s00159-016-0093-y

[4] Russell, R.W., Soifer, B.T. and Willner, S.P. (1977) The 4 to 8 Micron Spectrum of NGC 7027. The Astrophysical Journal, 217, L149-L153. https://doi.org/10.1086/182559

[5] Knacke, R.F. (1977) Carbonaceous Compounds in Interstellar Dust. Nature, 269, 132-134. https://doi.org/10.1038/269132a0

[6] Duley, W.W. and Williams, D.A. (1981) The Infrared Spectrum of Interstellar Dust: Surface Functional Groups on Carbon. Monthly Notices of the Royal Astronomical Society, 196, 269-274. https://doi.org/10.1093/mnras/196.2.269

[7] Leger, A. and Puget, J.L. (1984) Identification of the "Unidentified" IR Emission Features of Interstellar Dust? Astronomy and Astrophysics, 500, 279.

[8] Allamandola, L.J., Tielens, A.G.G.M. and Barker, J.R. (1985) Polycyclic Aromatic Hydrocarbons and the Unidentified Infrared Emission Bands-Auto Exhaust along the Milky Way. The Astrophysical Journal, 290, L25. https://doi.org/10.1086/184435

[9] Allamandola, L.J., Tielens, A.G.G.M. and Barker, J.R. (1989) Interstellar Polycyclic Aromatic Hydrocarbons-The Infrared Emission Bands, the Excitation/Emission Mechanism, and the Astrophysical Implications. The Astrophysical Journal Supplement Series, 71, 733. https://doi.org/10.1086/191396

[10] Simpson, J.P., Witteborn, F.C., Cohen, M. and Price, S.D. (1998) The Mid-Infrared Spectrum of the Galactic Center: A Starburst Nucleus. NASA, Washington DC. https://ntrs.nasa.gov/citations/19990008828

[11] Cesarsky, D., Jones, A.P., Lequeux, J. and Verstraete, L. (2000) Silicate Emission in Orion.

[12] Tielens, A.G.G.M. (2008) Interstellar Polycyclic Aromatic Hydrocarbon Molecules. Space Sciences Division, NASA Ames Research Center, Moffett Field.

[13] Kwok, S. and Zhang, Y. (2013) Unidentified Infrared Emission Bands: PAHs or MAONs? The Astrophysical Journal, 771, 5.

https://iopscience.iop.org/article/10.1088/0004-637X/771/1/5 https://doi.org/10.1088/0004-637X/771/1/5

[14] Netchitailo, V. (2019) Solar System. Angular Momentum. New Physics. Journal of High Energy Physics, Gravitation and Cosmology, 5, 112-139. https://doi.org/10.1103/PhysRevLett.39.165

[15] Lee, B.W. and Weinberg, S. (1977) Cosmological Lower Bound on Heavy-Neutrino Masses. Physical Review Letters, 39, 165-168. https://doi.org/10.1103/PhysRevLett.39.168 https://journals.aps.org/prl/abstract/10.1103/PhysRevLett.39.165

[16] Dicus, D.A., Kolb, E.W. and Teplitz, V.L. (1977) Cosmological Upper Bound on Heavy-Neutrino Lifetimes. Physical Review Letters, 39, 168-171. https://doi.org/10.1086/156031

[17] Dicus, D.A., Kolb, E.W. and Teplitz, V.L. (1978) Cosmological Implications of Massive, Unstable Neutrinos. The Astrophysical Journal, 221, 327-341. https://doi.org/10.1086/156335 
[18] Gunn, J.E., et al. (1978) Some Astrophysical Consequences of the Existence of a Heavy Stable Neutral Lepton. The Astrophysical Journal, 223, 1015-1031. https://doi.org/10.1086/156336

[19] Stecker, F.W. (1978) The Cosmic Gamma-Ray Background from the Annihilation of Primordial Stable Neutral Heavy Leptons. The Astrophysical Journal, 223, 1032-1036.

[20] Zeldovich, Y.B., Klypin, A.A., Khlopov, M.Y. and Chechetkin, V.M. (1980) Astrophysical Constraints on the Mass of Heavy Stable Neutral Leptons. Soviet Journal of Nuclear Physics, 31, 664-669. https://doi.org/10.4236/jhepgc.2019.51005

[21] Netchitailo, V. (2015) 5D World-Universe Model. Multicomponent Dark Matter. Journal of High Energy Physics, Gravitation and Cosmology, 1, 55-71. https://doi.org/10.4236/jhepgc.2015.12006

[22] (2021) What Are Galaxies? Cool Cosmos. https://coolcosmos.ipac.caltech.edu/ask/216-What-are-galaxies

[23] Ore, A. and Powell, J.L. (1949) Three-Photon Annihilation of an Electron-Positron Pair. Physical Review, 75, 1696. https://doi.org/10.1103/PhysRev.75.1696 https://journals.aps.org/pr/abstract/10.1103/PhysRev.75.1696 